\title{
Front Matter: Volume 7356
}

, "Front Matter: Volume 7356," Proc. SPIE 7356, Optical Sensors 2009, 735601 (23 June 2009); doi: 10.1117/12.834857

SPIE. Event: SPIE Optics + Optoelectronics, 2009, Prague, Czech Republic 


\title{
PROCEEDINGS OF SPIE
}

\section{Optical Sensors 2009}

\author{
Francesco Baldini \\ Jirí Homola \\ Robert A. Lieberman \\ Editors
}

\section{0-22 April 2009}

Prague, Czech Republic

Sponsored by

SPIE Europe

Cooperating Organizations

Institute of Physics, Academy of Sciences (Czech Republic)

Department of X-Ray Lasers, Institute of Physics (Czech Republic)

Czech and Slovak Society for Photonics

Photonics Society of Poland (Poland)

Published by

SPIE

Volume 7356 
The papers included in this volume were part of the technical conference cited on the cover and title page. Papers were selected and subject to review by the editors and conference program committee. Some conference presentations may not be available for publication. The papers published in these proceedings reflect the work and thoughts of the authors and are published herein as submitted. The publisher is not responsible for the validity of the information or for any outcomes resulting from reliance thereon.

Please use the following format to cite material from this book:

Author(s), "Title of Paper," in Optical Sensors 2009, edited by Francesco Baldini, Jiri Homola, Robert A. Lieberman, Proceedings of SPIE Vol. 7356 (SPIE, Bellingham, WA, 2009) Article CID Number.

ISSN 0277-786X

ISBN 9780819476302

Published by

SPIE

P.O. Box 10, Bellingham, Washington 98227-0010 USA

Telephone +1 3606763290 (Pacific Time) · Fax +1 3606471445

SPIE.org

Copyright (c) 2009, Society of Photo-Optical Instrumentation Engineers

Copying of material in this book for internal or personal use, or for the internal or personal use of specific clients, beyond the fair use provisions granted by the U.S. Copyright Law is authorized by SPIE subject to payment of copying fees. The Transactional Reporting Service base fee for this volume is $\$ 18.00$ per article (or portion thereof), which should be paid directly to the Copyright Clearance Center (CCC), 222 Rosewood Drive, Danvers, MA 01923. Payment may also be made electronically through CCC Online at copyright.com. Other copying for republication, resale, advertising or promotion, or any form of systematic or multiple reproduction of any material in this book is prohibited except with permission in writing from the publisher. The CCC fee code is 0277-786X/09/\$18.00.

Printed in the United States of America.

Publication of record for individual papers is online in the SPIE Digital Library.

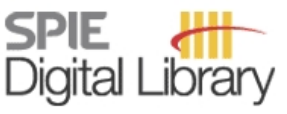

SPIEDigitalLibrary.org

Paper Numbering: Proceedings of SPIE follow an e-First publication model, with papers published first online and then in print and on CD-ROM. Papers are published as they are submitted and meet publication criteria. A unique, consistent, permanent citation identifier (CID) number is assigned to each article at the time of the first publication. Utilization of CIDs allows articles to be fully citable as soon they are published online, and connects the same identifier to all online, print, and electronic versions of the publication. SPIE uses a six-digit CID article numbering system in which:

- The first four digits correspond to the SPIE volume number.

- The last two digits indicate publication order within the volume using a Base 36 numbering system employing both numerals and letters. These two-number sets start with 00, 01, 02, 03, 04 , $05,06,07,08,09,0 A, 0 B \ldots$. OZ, followed by 10-1Z, 20-2Z, etc.

The CID number appears on each page of the manuscript. The complete citation is used on the first page, and an abbreviated version on subsequent pages. Numbers in the index correspond to the last two digits of the six-digit CID number. 


\section{Contents}

xi Conference Committee

xiii Photon physics: from wave mechanics to quantum electrodynamics (Plenary Paper) [7355-100]

O. Keller, Aalborg Univ. (Denmark)

\section{SESSION 1 ADVANCES IN TRANSDUCERS}

735602 Optical fingerprinting with computer screen photo-assisted techniques (Invited Paper) [7356-01]

D. Filippini, I. Lundström, Linköping Univ. (Sweden)

735603 Label-free biosensing by means of optical micro-ring resonator [7356-02]

M. Iodice, L. De Stefano, G. Coppola, V. Mocella, I. Rea, E. De Tommasi, E. Orabona, I. Rendina, Institute for Microelectronics and Microsystems (Italy)

735604 Microchanneled chirped fibre Bragg gratings for simultaneous refractive index and temperature measurements [7356-03]

P. Saffari, Aston Univ. (United Kingdom); H. Fu, Zhejiang Univ. (China); K. Zhou, L. Zhang,

I. Bennion, Aston Univ. (United Kingdom)

735605 Analysis and modeling of a silicon nitride slot-waveguide microring resonator biochemical sensor [7356-04]

C. A. Barrios, Univ. Politécnica de Madrid (Spain)

735606 Spin coating and plasma process for 2.5D and hybrid 3D micro-resonators on multilayer polymers [7356-05]

B. Bêche, Institut de Physique de Rennes, CNRS, Univ. de Rennes I (France); E. Gaviot, Lab. d'Acoustique de l'Univ. du Maine, CNRS (France); C. Godet, A. Zebda, A. Potel, J. Barbe, Institut de Physique de Rennes, CNRS, Univ. de Rennes I (France); L. Camberlein, Lab. d'Acoustique de l'Univ. du Maine, CNRS (France); V. Vié, P. Panizza, G. Loas, C. Hamel, Institut de Physique de Rennes, CNRS, Univ. de Rennes I (France); J. Zyss, IFR d'Alembert, Lab. de Photonique Quantique et Moléculair, ENS Cachan, CNRS (France); N. Huby, Institut de Physique de Rennes, CNRS, Univ. de Rennes I (France)

\section{SESSION 2 INTERFEROMETRIC SENSORS}

735607 Refractometric sensor based on all-fiber coaxial Michelson interferometers [7356-07] P. Barrios, Univ. Autónoma de San Luis Potosí (Mexico); D. Sáez-Rodríguez, Univ. de València (Spain); A. Rodríguez, Univ. Autónoma de San Luis Potosí (Mexico); J. L. Cruz, A. Díez, M. V. Andrés, Univ. de València (Spain)

735608 Laser-self-mixing interferometric fiber strain sensor [7356-47]

A. Intermite, B. Radisavljevic, S. Ottonelli, M. Dabbicco, G. Scamarcio, CNR-INFM Lab. Regionale LIT3 (Italy) and Univ. degli Studi di Bari (Italy) 
7356 OC Development of portable SPR sensor devices based on integrated periodic arrays of nanoholes [7356-12]

F. Eftekhari, J. Ferreira, M. L. J. Santos, C. Escobedo, A. G. Brolo, D. Sinton, R. Gordon, Univ. of Victoria (Canada)

7356 OD Surface plasmon resonance imaging for parallelized detection of protein biomarkers [7356-13]

M. Piliarik, L. Párová, H. Vaisocherová, J. Homola, Institute of Photonics and Electronics (Czech Republic)

7356 OE Visualization of surface electromagnetic waves in one-dimensional photonic crystal by fluorescence dye [7356-14]

I. V. Soboleva, M.V. Lomonosov Moscow State Univ. (Russian Federation); E. Descrovi, Politecnico di Torino (Italy); L. Dominici, F. Michelotti, Univ. degli Studi di Roma, La Sapienza (Italy); F. Giorgis, Politecnico di Torino (Italy); A. A. Fedyanin, M.V. Lomonosov Moscow State Univ. (Russian Federation)

\section{SESSION 4 PHYSICAL SENSORS I}

7356 OG Ambient humidity monitoring using a 1D photonic crystal sensor fabricated with glancing angle deposition [7356-16]

M. M. Hawkeye, K. M. Krause, Univ. of Alberta (Canada); M. J. Brett, Univ. of Alberta

(Canada) and National Institute for Nanotechnology (Canada)

7356 ol Miniature laser doppler velocimetry systems [7356-18]

C. I. Moir, Exact Group LLP (United Kingdom)

\section{SESSION 5 PHYSICAL SENSORS II}

7356 OK Influence of residual fiber birefringence and temperature on the high-current performance of an interferometric fiber-optic current sensor [7356-20]

R. Wüest, A. Frank, S. Wiesendanger, P. Gabus, U. E. Meier, J. Nehring, K. Bohnert, ABB Ltd. (Switzerland)

$7356 \mathrm{OL}$ Pump depletion reduction technique for extended-range distributed Brillouin fiber sensors [7356-21]

R. Bernini, IREA, Consiglio Nazionale delle Ricerche (Italy); A. Minardo, L. Zeni, Second Univ. of Naples (Italy)

7356 ON On the minimization of timing walk in industrial pulsed time-of-flight laser radars [7356-23] J. Kostamovaara, J. Nissinen, S. Kurtti, I. Nissinen, J. Jansson, A. Mäntyniemi, Univ. of Oulu (Finland) 
735600 All-silicon carbide hybrid wireless-wired optics temperature sensor: turbine tests and distributed fiber sensor network design [7356-24]

N. A. Riza, M. Sheikh, College of Optics and Photonics, Univ. of Central Florida (United States)

7356 OP Laser light-section sensor automating the production of textile-reinforced composites [7356-25]

R. Schmitt, C. Niggemann, C. Mersmann, RWTH Aachen Univ. (Germany)

$7356 \mathrm{OQ}$ A self-mixing laser sensor for the real-time correction of straightness/flatness deviations of a linear slide [7356-26]

S. Ottonelli, M. Dabbicco, F. De Lucia, G. Scamarcio, CNR-INFM Lab. Regionale LIT3 (Italy) and Univ. degli Studi di Bari (Italy)

$7356 \mathrm{OR}$ Optical sensing of magnetic field based on magnetorefractive effect in manganites [7356-27]

D. Hrabovský, Technical Univ. of Ostrava (Czech Republic); G. Herranz, Institut de Ciéncia de Materials de Barcelona (Spain); K. Postava, Technical Univ. of Ostrava (Czech Republic); I. C. Infante, F. Sánchez, J. Fontcuberta, Institut de Ciéncia de Materials de Barcelona (Spain)

7356 OS Optical system for the simultaneous measurement of two-dimensional straightness errors and the roll angle [7356-28]

I. Rahneberg, H.-J. Büchner, G. Jäger, Technische Univ. IImenau (Germany)

7356 ОТ Optical sensing in laser machining [7356-29]

I. Smurov, M. Doubenskaia, Ecole Nationale d'Ingénieurs de Saint-Etienne (France)

\section{SESSION 7 COMPONENTS FOR SENSORS}

7356 OV Slab waveguide spatial heterodyne spectrometers for remote sensing from space [7356-31] M. Florjańczyk, York Univ. (Canada) and National Research Council Canada (Canada); P. Cheben, S. Janz, B. Lamontagne, J. Lapointe, National Research Council Canada (Canada); A. Scott, York Univ. (Canada) and COM DEV Ltd. (Canada); B. Solheim, York Univ. (Canada); D.-X. Xu, National Research Council Canada (Canada)

7356 OW Organic photo sensors operating at high speed utilizing poly(9,9-dioctylfluorene) derivative and fullerene derivative fabricated by solution process [7356-32]

Y. Ohmori, T. Hamasaki, H. Kajii, Osaka Univ. (Japan); T. Morimune, Takuma National College of Technology (Japan)

7356 0X Sensitivity of fast-response nanographite photodetector at high temperature [7356-33]

G. M. Mikheev, R. G. Zonov, Institute of Applied Mechanics (Russian Federation); A. N. Obraztsov, Moscow State Univ. (Russian Federation); Y. P. Svirko, Joensuu Univ. (Finland) 
7356 OY Remote tuneable diode laser spectroscopy (TDLS) via a IW Raman source [7356-34]

D. Mitchell, K. Duffin, W. Johnstone, Univ. of Strathclyde (United Kingdom)

$73560 Z$ Chemical sensor applications of whispering-gallery modes resonances of thin capillaries with submicrometric wall [7356-35]

V. Zamora, A. Díez, M. V. Andrés, B. Gimeno, Univ. de Valencia (Spain)

735610 Infrared optical sensor for $\mathrm{CO}_{2}$ detection [7356-36]

F. Charpentier, V. Nazabal, J. Troles, Q. Coulombier, L. Brilland, C. Boussard-Plédel, Equipe Verres et Céramiques, CNRS, Sciences chimiques de Rennes, Univ. de Rennes 1 (France); P. Nemec, Univ. of Pardubice (Czech Republic); H. Lhermite, Univ. de Rennes 1 (France); J. Charrier, FOTON-CCLO, Univ. de Rennes 1 (France); F. Smektala, Institut Carnot de Bourgogne, CNRS, Equipe Solitons Lasers et Communications Optiques, Univ. de Bourgogne (France); M. Frumar, Univ. of Pardubice (Czech Republic); K. Le Pierres, BRGM (France); N. Thybaud, ADEME (France); B. Bureau, Equipe Verres et Céramiques, CNRS, Sciences chimiques de Rennes, Univ. de Rennes 1 (France)

$735611 \quad$ Using liquid crystals as optical gas sensors to detect thiol vapors [7356-38] H. XU, K.-L. Yang, National Univ. of Singapore (Singapore)

\section{SESSION 9 BIOSENSORS}

735612 Principles and applications of fluorescence lifetime correlation spectroscopy (Invited Paper) [7356-39]

L. Beranová, J. Humpolíčková, M. Hof, J. Heyrovský Institute of Physical Chemistry (Czech Republic)

735613 A fluorescent immunoassay for the determination of procalcitonin and C-reactive protein [7356-40]

F. Baldini, Institute of Applied Physics (Italy); L. Bolzoni, Datamed S.r.L. (Italy); A. Giannetti, Institute of Applied Physics (Italy); G. Porro, Datamed S.r.L. (Italy); F. Senesi, C. Trono, Institute of Applied Physics (Italy)

735615 Intrinsic photoluminescence of diatom shells in sensing applications [7356-42]

E. De Tommasi, I. Rendina, I. Rea, Institute for Microelectronics and Microsystems (Italy); M. De Stefano, A. Lamberti, Univ. of Naples Federico II (Italy); L. De Stefano, Institute for Microelectronics and Microsystems (Italy)

735616 Perfluorinated polymer optical fiber tapers for fluorescence collection [7356-43]

R. Gravina, G. Testa, R. Bernini, IREA-CNR (Italy)

\section{POSTER SESSION}

735617 Toward extended range sub-micron conoscopic holography profilometers using multiple wavelengths and phase measurement [7356-44]

J. M. Enguita, I. Álvarez, Univ. of Oviedo (Spain); J. Marina, DSIPlus (Spain); G. Ojea,

J. A. Cancelas, M. Frade, Univ. of Oviedo (Spain) 
735618 Measuring small thickness changes of a thin film by white-light spectral interferometry [7356-45]

P. Hlubina, J. Luňáček, D. Ciprian, M. Luňáčková, Technical Univ. of Ostrava (Czech Republic)

735619 A signal-to-noise ratio comparison of high dynamic range CMOS image sensors [7356-48] L. H. C. Braga, S. Domingues, J. G. Gomes, A. C. Mesquita, Univ. Federal do Rio de Janeiro (Brazil)

7356 1B Application of a silica-on-silicon planar optical waveguide Bragg grating sensor for organic liquid compound detection [7356-51]

S. Scheurich, S. Belle, R. Hellmann, Univ. of Applied Sciences (Germany); S. So,

I. J. G. Sparrow, G. Emmerson, Stratophase Ltd. (United Kingdom)

7356 ID Preparation of $\mathrm{Ni} / \mathrm{Zn}$ and $\mathrm{NiO} / \mathrm{ZnO}$ heterojunction nanowires and their optoelectrical characteristics [7356-53]

W.-C. Tsai, S.-J. Wang, C.-R. Tseng, R.-M. Ko, National Cheng Kung Univ. (Taiwan); J.-C. Lin, St. John's Univ. (Taiwan)

$7356 \mathrm{IF}$ Preparation and optoelectronic properties of NiO/ZnO heterostructure nanowires [7356-55] W.-C. Tsai, S.-J. Wang, C.-R. Tseng, R.-M. Ko, National Cheng Kung Univ. (Taiwan); J.-C. Lin, St. John's Univ. (Taiwan)

$73561 \mathrm{G} \quad$ Novel optical-fiber structure as a tension sensor [7356-56]

J. Skapa, P. Siska, V. Vasinek, J. Vanda, Technická univ. Ostrava (Czech Republic)

735611 Photo-erasure of type-II femtosecond laser written Bragg gratings employed as high reflectors in moderate power Q-switch fibre laser [7356-58]

M. L. Åslund, The Univ. of Sydney (Australia); N. Jovanovic, Macquarie Univ. (Australia);

S. D. Jackson, J. Canning, The Univ. of Sydney (Australia); G. D. Marshall, A. Fuerbach, M. J. Withford, Macquarie Univ. (Australia); K. Cook, The Univ. of Sydney (Australia)

7356 i J Compact all-fiber light source for Brillouin sensor applications [7356-59]

C. Cuadrado-Laborde, Univ. de València (Spain) and Ctr. de Investigaciones Ópticas (Argentina); P. Pérez-Millán, M. V. Andrés, A. Díez, J. L. Cruz, Univ. de València (Spain); Y. O. Barmenkov, Ctr. de Investigaciones en Óptica (Mexico)

7356 1K Dew point measurement technique utilizing fiber cut reflection [7356-60] S. M. Kostritskii, A. A. Dikevich, Y. N. Korkishko, V. A. Fedorov, Optolink Ltd., Moscow Institute of Electronic Technology (Russian Federation)

$73561 \mathrm{~L} \quad$ Air-suspended solid-core fibers for sensing [7356-61] S. Selleri, E. Coscelli, M. Sozzi, A. Cucinotta, F. Poli, D. Passaro, Univ. degli Studi di Parma (Italy)

$73561 \mathrm{M}$ Usage of liquid crystals in optical sensors of mechanical forces and motion [7356-62] S. V. Pasechnik, Moscow State Univ. of Instrument Engineering and Computer Science (Russian Federation) and Hong Kong Univ. of Science and Technology (Hong Kong, China); D. V. Shmeliova, V. A. Tsvetkov, A. V. Torchinskaya, Moscow State Univ. of Instrument Engineering and Computer Science (Russian Federation); V. G. Chigrinov, Hong Kong Univ. of Science and Technology (Hong Kong, China) 
7356 iN Ratiometric wavelength monitor based on X-type spectral response using two edge filters [7356-63]

A. M. Hatta, G. Rajan, G. Farrell, Y. Semenova, Dublin Institute of Technology (Ireland)

735610 Experimental demonstration of a ferroelectric liquid crystal tunable filter for fast demodulation of FBG sensors [7356-64]

S. Mathews, Y. Semenova, G. Rajan, G. Farrell, Dublin Institute of Technology (Ireland)

7356 IR Numerical analysis of reflection characteristics of cascaded non-uniform fiber Bragg gratings [7356-67]

E. Gemzický, J. Müllerová, Univ. of Žilina (Slovakia)

7356 is Multi-wavelength switchable fibre ring laser based on polarisation selective tilted fibre gratings capable of strain and temperature sensing [7356-68]

P. Saffari, C. Mou, Aston Univ. (United Kingdom); H. Fu, Zhejiang Univ. (China); L. Zhang,

I. Bennion, Aston Univ. (United Kingdom)

7356 1T Performance analysis of diode optopair gas sensors [7356-69]

G. Y. Sotnikova, S. E. Aleksandrov, G. A. Gavrilov, Ioffe Physical-Technical Institute (Russian Federation)

$73561 \mathrm{U} \quad$ Local real-time detection of $\mathrm{pH}$ using fibre tapers [7356-70]

I. Kasik, T. Martan, O. Podrazky, J. Mrazek, M. Pospisilova, V. Matejec, Institute of Photonics and Electronics (Czech Republic)

7356 1X Frequency fiber-optical sensor system with wavelength division multiplexing [7356-73] A. V. Poliakov, Belarusian State Univ. (Belarus)

7356 1Y Optical fiber sensor based on redistribution of power among several guided modes [7356-74]

P. Siska, J. Skapa, V. Vasinek, F. Hanacek, Technical Univ. of Ostrava (Czech Republic);

I. Kasik, Institute of Photonics and Electronics (Czech Republic)

735612 Design and evaluation of diffractive optical elements: optimization by using iterative angular spectrum approach and evaluation based on vector diffraction theory [7356-75] S. Yoshida, M. Yamamoto, Tokyo Univ. of Science (Japan)

735620 Observation of plasmon-induced optical field enhancement near a pair of partially metal covered dielectric spheres manipulated by optical tweezers [7356-77]

A. G. Zhdanov, A. A. Fedyanin, M.V. Lomonosov Moscow State Univ. (Russian Federation);

S. Rao, M. Kreuzer, S. Ballint, D. Petrov, ICFO-Institute of Photonic Science (Spain)

$735621 \quad$ Micro force measurement by an optical method [7356-78]

N. Khélifa, Conservatoire National des Arts et Métiers (France)

735622 Sensitivity enhancement in surface plasmon resonance sensors: theoretical modeling [7356-79]

J. Vlček, J. Pištora, M. Lesňák, Technical Univ. of Ostrava (Czech Republic) 
735624 Characterization of sensing layer onto the tip tapered fiber [7356-81]

M. Pospisilova, Institute of Photonics and Electronics (Czech Republic); J. Petrasek, Institute of Experimental Botany (Czech Republic); V. Matejec, I. Kasik, Institute of Photonics and Electronics (Czech Republic)

735625 Biosensor platform based on surface plasmon-enhanced fluorescence spectroscopy and responsive hydrogel binding matrix [7356-82]

C.-J. Huang, Austrian Research Ctrs. (Austria); U. Jonas, Max-Planck Institute for Polymer Research (Germany) and FORTH/IESL (Greece); J. Dostálek, W. Knoll, Austrian Research Ctrs. (Austria)

735627 Experimental performances and Monte Carlo modelling of LWIR HgCdTe avalanche photodiodes [7356-84]

S. Derelle, S. Bernhardt, R. Haidar, J. Deschamps, J. Primot, ONERA/DOTA (France);

J. Rothman, CEA/LETI (France); S. Rommelvere, N. Guérineau, ONERA/DOTA (France)

735628 Sensor of back-scattered light polarization in body cells [7356-85]

P. Tománek, J. Mikláš, A. Bajgar, L. Grmela, P. Dobis, J. Brüstlová, Brno Univ. of Technology (Czech Republic)

7356 2A Further developments on a novel color sensitive CMOS detector [7356-89]

G. Langfelder, A. Longoni, F. Zaraga, Politecnico di Milano (Italy)

7356 2C Broadband transmission spectroscopy in tissue: application to radiofrequency tissue fusion [7356-91]

T. Floume, R. R. A. Syms, Imperial College London (United Kingdom); A. W. Darzi, G. B. Hanna, St. Mary's Hospital, Imperial College London (United Kingdom)

Author Index 
Downloaded From: https://www.spiedigitallibrary.org/conference-proceedings-of-spie on 26 Apr 2023

Terms of Use: https://www.spiedigitallibrary.org/terms-of-use 


\title{
Conference Committee
}

\author{
Symposium Chairs
}

Pavel Tomanek, Brno University of Technology (Czech Republic)

Alan Michette, King's College London (United Kingdom)

Bahaa Saleh, Boston University (United States)

Symposium Honorary Chair

Jan Perina, Sr., Palacký University (Czech Republic)

Conference Chairs

Francesco Baldini, Institute of Applied Physics, CNR (Italy)

Jirí Homola, Institute of Photonics and Electronics (Czech Republic)

Robert A. Lieberman, Intelligent Optical Systems, Inc. (United States)

Program Committee

Vladimir Baumruk, Charles University in Prague (Czech Republic)

Artur Dybko, Warsaw University of Technology (Poland)

N. Jaffrezic, Centre de Génie Electrique de Lyon (France)

Laura Maria Lechuga, CIN2 Research Center on Nanoscience and Nanotechnology (Spain)

Bo Liedberg, Linköpings Universitet (Sweden)

Aleksandra Lobnik, University of Maribor (Slovenia)

Ramaier Narayanaswamy, The University of Manchester (United Kingdom)

Guillermo Orellana, Universidad Complutense de Madrid (Spain)

Reinhardt Willsch, IPHT Jena (Germany)

Otto S. Wolfbeis, Universität Regensburg (Germany)

\section{Session Chairs}

1 Advances in Transducers

Jiří Homola, Institute of Photonics and Electronics (Czech Republic)

2 Interferometric Sensors

Jiří Homola, Institute of Photonics and Electronics (Czech Republic)

3 SPR Sensors

Ingemar Lundström, Linköpings Universitet (Sweden) 
4 Physical Sensors I

Francesco Baldini, Institute of Applied Physics, CNR (Italy)

5 Physical Sensors II

Francesco Baldini, Institute of Applied Physics, CNR (Italy)

6 Physical Sensors III

Ivan Kasik, Institute of Photonics and Electronics (Czech Republic)

7 Components for Sensors

Ivan Kasik, Institute of Photonics and Electronics (Czech Republic)

8 Chemical Sensors

Martin Hof, J. Heyrovský Institute of Physical Chemistry (Czech Republic)

9 Biosensors

Jiří Homola, Institute of Photonics and Electronics (Czech Republic) 